\title{
Kits19 segmentation challenge
}

\author{
Ilyas Sirazitdinov $^{1}$ and Bulat Ibragimov ${ }^{1}$ \\ Innopolis University, Innopolis, Respublic Tatarstan 420500, Russian Federation \\ i.sirazitdinov@innopolis.ru
}

\begin{abstract}
In this work we briefly describe our methodology for the kidney segmentation problem.
\end{abstract}

Keywords: Kits19

\section{Methodology}

\subsection{Data preprocessing}

As it was proposed by authors of the challenge, we zoomed all data to the same scale. After we analyzed the size distributions of kidney and tumor we additionally scaled all images by factor 2 . As a result, $90 \%$ percentile of kidney size became equal to the batch size. All images were clipped to the range ($1000 ; 1000)$ and normalized by mean subtraction and standard deviation division. Mask channels were augmented with their contours. The dataset was split into 5 -folds with equal distribution of kidney size in each fold.

Model In our work, we used a U-net based model with a ResNet encoder. The final layer consists of 4 channels ( 2 for masks and 2 for contours) with a sigmoid activation function.

Training To train our model we used AdamW optimizer with weight decay. We picked a cosine annealing policy for the learning rate scheduling. During the first cycles, we applied affine and color augmentation of images. The batch size $[48,96,96]$ was able to provide enough context for the image. We used a weighted loss: binary cross-entropy and soft dice.

Prediction During the prediction the same preprocessing was applied to the test images. We used a patch-wise strategy with overlapping for the prediction. The results of inference from best 5 -folds models were averaged and rounded by thresholds. 